Wien klin Mag 2019 $22: 53$

https://doi.org/10.1007/s00740-019-0280-3

(c) Springer-Verlag GmbH Austria, ein Teil von Springer Nature 2019

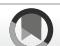

Verena Kienast

SpringerMedizin, Wien, Österreich

\title{
Die Dissonanz - eine Frage der Perspektive
}

\section{Ein schmaler Grat zwischen Freude, Pflicht und Belastung}

viele Arbeitnehmer von einem Urlaub zum nächsten. Anfängliche Begeisterung im Beruf weicht bei einer beträchtlichen Anzahl der Erwachsenen bedauerlicherweise zunehmender Frustration, Überlastung, Ernüchterung. Flexiblen Arbeits- und Freizeitregelungen steht das sattsam bekannte Beharrungsvermögen der Entscheidungsverantwortlichen gegenüber. Von der Politik kommen dazu Worthülsen, die das Nichts maskieren: Wir wollen, so Kanzleramtsminister Gernot Blümel zur zunächst beabsichtigten Halbtagsregel des Karfreitag, „dass sich möglichst wenig ändert, ... Mehrbelastungen für die Wirtschaft sind schwer $\mathrm{zu}$ rechtfertigen, ... gleichzeitig wollen wir, dass den Leuten, die betroffen sind, möglichst nichts weggenommen wird.“ Kreative Zukunftsgestaltung sieht anders aus. Die nun beschlossene Lösung gehört auch nicht dazu. Freude an der Arbeit haben übrigens überwiegend jene aus den Bürgerkriegsgebieten im Nahen Osten geflüchteten Mediziner, die mittlerweile durchaus zahlreich ihr Medizinstudium nostrifizieren lassen konnten und in österreichischen Spitälern arbeiten. Freude mit diesen Medizinern haben auch die Patienten, weil das Engagement der neuen Ärzte nach der langen und anstrengenden Berufsanerkennung sehr hoch ist. Es ist $\mathrm{zu}$ hoffen, dass dies so bleibt.

\section{Duales westliches Denken}

Nicht viel anders geht es im späteren Alter: Sind zwar die meisten Menschen eher froh, nicht arbeitslos zu sein, ist der Ruf nach einem zusätzlichen Feiertag zuletzt durch die Karfreitagsdiskussion wieder laut geworden und quälen sich

Erstaunlich dissonant stellt sich das Handeln des Staats bzw. von dessen Akteuren in unterschiedlichsten Zusammenhängen dar. Die Frage, was mit den österreichischen Staatsbürgern, die geglaubt haben, sich dem „islamischen Staat“ in Syrien oder dem Irak anschließen zu müssen und nun dort in Gefangenschaft sind, hat der Internationale Haftbefehl, den das österreichische Justizministerium für manche dieser Personen ausgestellt haben soll, mehr oder weniger unbeabsichtigt auf juristischer Ebene beantwortet. Hier wird es wohl auch darum geben, Staatsverantwortung zu übernehmen.

Dass derart widersprüchliche Aktionen bei uns oft als Zwiespältigkeit empfunden wird, ist freilich auch Ausdruck des dualen westlichen Denkens, mit dem sich die asiatischen Philosophien meist nicht herumschlagen müssen: Denn es geht um das Eine und dazu gehören eben alle - auch die widersprüchlichen - Teile.

meint Ihre<smiles>CCCC1CCCCC1C</smiles>

V. Kienast

\section{Korrespondenzadresse}

Verena Kienast

SpringerMedizin

Wien, Österreich

Verena.kienast@springer.at
Hinweis des Verlags. Der Verlag bleibt in Hinblick auf geografische Zuordnungen und Gebietsbezeichnungen in veröffentlichten Karten und Institutsadressen neutral. 